\title{
Dynamics of relaxor ferroelectrics
}

\author{
R. Pirc, R. Blinc, and V. Bobnar \\ Jožef Stefan Institute, P.O. Box 3000, 1001 Ljubljana, Slovenia
}

(October 25, 2018)

\begin{abstract}
We study a dynamic model of relaxor ferroelectrics based on the spherical random-bond - random-field model and the Langevin equations of motion written in the representation of eigenstates of the random interaction matrix. The solution to these equations is obtained in the long-time limit where the system reaches an equilibrium state in the presence of random local electric fields. The complex dynamic linear and third-order nonlinear susceptibilities $\chi_{1}(\omega)$ and $\chi_{3}(\omega)$, respectively, are calculated as functions of frequency and temperature. In analogy with the static case, the dynamic model predicts a narrow frequency dependent peak in $\chi_{3}(T, \omega)$, which mimics a transition into a glass-like state, but a real transition never occurs in the case of non-zero random fields. A freezing transition can be described by introducing the empirical Vogel-Fulcher (VF) behavior of the relaxation time $\tau$ in the equations of motion, with the $\mathrm{VF}$ temperature $T_{0}$ playing the role of the freezing temperature $T_{f}$. The scaled third-order nonlinear susceptibility $a_{3}^{\prime}(T, \omega)=\bar{\chi}_{3}^{\prime}(\omega) / \bar{\chi}_{1}^{\prime}(3 \omega) \bar{\chi}_{1}^{\prime}(\omega)^{3}$, where the bar denotes a statistical average over $T_{0}$, shows a crossover from paraelectric-like to glass-like behavior in the quasistatic regime above $T_{f}$. The shape of $\bar{\chi}_{1}(\omega)$ and $\bar{\chi}_{3}(\omega)$-and thus of $a_{3}^{\prime}(T, \omega)$ - depends crucially on the probability distribution of $\tau$. It is shown that for a linear distribution of VF temperatures $T_{0}, a_{3}^{\prime}(T, \omega)$ has a peak near $T_{f}$ and shows a strong frequency dispersion in the low temperature region.
\end{abstract}

PACS numbers:77.84.Dy, 64.70.Pf, 77.22.-d 


\section{INTRODUCTION}

Relaxor ferroelectrics (or relaxors) represent a new low-temperature state of polar dielectrics, which can be regarded as an intermediate state between dipolar glasses and normal ferroelectrics [1,2]. Some of the concepts developed for dipolar glasses, such as the EdwardsAnderson (EA) order parameter, are applicable to relaxors as well, as recently shown for $\mathrm{PbMg}_{1 / 3} \mathrm{Nb}_{2 / 4} \mathrm{O}_{3}(\mathrm{PMN})$ [3], $\mathrm{PbSc}_{1 / 2} \mathrm{Ta}_{1 / 2} \mathrm{O}_{3}$ (PST) [4], and $\mathrm{Pb}_{1-x} \mathrm{La}_{2 x / 3} \mathrm{Zr}_{y} \mathrm{Ti}_{1-y} \mathrm{O}_{3}(\mathrm{PLZT})$ [5]. In contrast to dipolar glasses, where elementary dipole moments exist on the atomic scale, the relaxor state is characterized by the presence of nanosized polar clusters of variable sizes. This picture constitutes the basis of the superparaelectric model [1] and of the more recent reorientable polar cluster model of relaxors [2,6]. By including explicitly the long-range frustrated intercluster interactions of a spin glass type into this picture, one arrives at the so-called spherical random-bond-random-field (SRBRF) model of relaxor ferroelectrics [7], which is capable of describing the static behavior of relaxors, such as the line shape of quadrupole perturbed NMR in PMN [3] and PST [4], and the sharp increase of the quasistatic third-order nonlinear dielectric constant [3,5].

The unusually large value of the static linear dielectric permittivity can also be explained within the framework of the SRBRF model if one assumes that the mean value of the random coupling $J_{0}$ is very close to-but slightly smaller than-its r.m.s. variance $J$, whereas in dipolar glasses the latter is usually dominant. By including an ad hoc electric field dependence of $J_{0}$ into the model, one can furthermore describe the transition from the relaxor to an inhomogeneous ferroelectric state for fields $E$ exceeding a critical value $E_{c}$ [8]. It should be noted, however, that the random electric fields, which exist both in dipolar glasses [10] and in relaxors [2,6, 9], seem to be much weaker in the latter case. It is interesting to note that Korner et. al [11] have reported a relaxor phase in the dipolar glass DRADP in a narrow range of concentration just above the dipolar glass phase, where the system is expected to behave as an inhomogeneous antiferroelectric.

While the static SRBRF model describes a relaxor system in thermodynamic equilibrium, there are a number of phenomena suggesting that relaxors, in particular their low temperature state, are dominated by nonequilibrium effects. Typical examples are the difference between the field-cooled and zero-field cooled static dielectric constant, and the occurrence of strong frequency dispersion in both the linear and nonlinear dielectric permittivity at low temperatures. Is is clear that these properties can only be discussed within a dynamic model. In the present paper, we introduce a dynamic model, which an extension of the SRBRF model to dynamic problems. Following Vugmeister and Rabitz [2,6] we assume that polar clusters can reorient with a characteristic relaxation time $\tau$ and write down the corresponding Langevin equations of motion, which are based on the static SRBRF Hamiltonian. These equations explicitly contain the frustrated interactions between the polar clusters and thus allow us to study the effects of these interactions on both the equilibrium and nonequilibrium properties. In particular, we will discuss here the anomalous temperature dependence of the nonlinear dielectric susceptibility and the crossover from the paraelectriclike to inhomogeneous ferroelectric-like behavior observed in PMN and PLZT [5]. As in spin glasses, the Langevin equations based on the spherical model can be solved exactly [12]; some additional features appear in view of the presence of random fields [13]. Here we will focus on the asymptotic solutions corresponding to equilibirum dynamics, such as observed 
in a typical dielectric relaxation experiment.

In Sec. II we introduce the uniaxial SRBRF model Hamiltonian in the representation of eigenstates of the random interaction and write down the Langevin equations of motion. The asymptotic solution is studied in Sec. III, where the static linear and nonlinear susceptibilities are derived. In Sec. IV the dynamic linear response is given, and in Sec. V the corresponding results for the third-order nonlinear response are derived. Finally, in Sec. VI we present our conclusions.

\section{DYNAMIC SRBRF MODEL}

In general, the polarization of $i$-th polar cluster, $i=1,2, \ldots, N$, is a three component $(n=3)$ vector $\vec{S}_{i}=\left(S_{i x}, S_{i y}, S_{i z}\right)$, its length being restricted solely by the spherical condition $\sum_{i}\left(\vec{S}_{i}\right)^{2}=3 N$. In the present work we will discuss the simpler uniaxial $(n=1)$ case $-\sqrt{N}<S_{i}<+\sqrt{N}$, where $S_{i}$ is subject to the spherical condition

$$
\sum_{i=1}^{N} S_{i}^{2}=N
$$

The SRBRF model Hamiltonian is thus

$$
\mathcal{H}=-\frac{1}{2} \sum_{i j} J_{i j} S_{i} S_{j}-\sum_{i} h_{i} S_{i}-g E \sum_{i} S_{i},
$$

where $J_{i j}$ are the randomly frustrated intercluster interactions, $h_{i}$ local random electric fields, $E$ an applied uniform electric field, and $g$ the appropriate dipole moment [7]. As usual, $J_{i j}$ is assumed to be infinitely ranged and distributed according to Gaussian statistics with average value $J_{0} / N$ and cumulant variance $J^{2} / N$. The Gaussian random fields $h_{i}$ are characterized by the random average

$$
\left[h_{i} h_{j}\right]_{a v}=\Delta \delta_{i j}
$$

The uniaxial SRBRF model (2) has potential applicability to uniaxial relaxors such as $\mathrm{Sr}_{1-x} \mathrm{Ba}_{x} \mathrm{Nb}_{2} \mathrm{O}_{6}(\mathrm{SBN})$. The present results can be, however, generalized to the isotropic $n=3$ case as long as there is no mixing of the $x, y, z$ components [7].

The Langevin equations of motion for the variables $S_{i}(t)$ are written as

$$
\tau \frac{\partial S_{i}(t)}{\partial t}=-\frac{\partial(\beta \mathcal{H})}{\partial S_{i}}-2 z(t) S_{i}(t)+\xi_{i}(t)
$$

Here $\tau$ is the characteristic relaxation time for the reorientation of polar clusters. Eq. (4) implies that $\tau$ is site independent, however, some variation of $\tau$ across the system should in principle not be excluded, resulting in a distribution of relaxation times [2, 6]. The function $z(t)$ plays the role of a Langrange multiplier enforcing the spherical condition (1) at all times [12].

The stochastic Langevin forces $\xi_{i}(t)$ ensure the proper equilibrium distribution and are determined by their ensemble averages 


$$
\left\langle\xi_{i}(t) \xi_{j}\left(t^{\prime}\right)\right\rangle_{a v}=2 \tau \delta_{i j} \delta\left(t-t^{\prime}\right) .
$$

Following the theory of spherical spin glasses we now transform to the representation of eigenstates $\psi_{\lambda}(k)$ and eigenvalues $J_{\lambda}$ of the random matrix $J_{i j}$ [14, 12, 13]. This is done in two steps [15]: First, one introduces "spin wave" states $S_{k}=N^{-1 / 2} \sum_{i} \exp \left(i k R_{i}\right) S_{i}$; next, these are expanded in normal modes

$$
S_{\lambda}=\sum_{k} \psi_{\lambda}(k) S_{k} .
$$

The transformed equation of motion (田) becomes explicitly

$$
\frac{\partial S_{\lambda}}{\partial t}=\beta\left[J_{\lambda}-2 z(t)\right] S_{\lambda}+\beta h_{\lambda} S_{\lambda}+g \Psi_{\lambda}(0) E(t) S_{\lambda}+\xi_{\lambda}(t) .
$$

Here $\Psi_{\lambda}(0)=N \psi_{\lambda}(0)$ and we have rescaled the time to a new dimensionless variable $t \rightarrow t / \tau$. Assuming a field $E(t)$ applied at $t=0$ and introducing the integrating factor

$$
\phi_{\lambda}(t)=\exp \left[\beta J_{\lambda} t-2 \int_{0}^{t} d t^{\prime} z\left(t^{\prime}\right)\right]
$$

we obtain the solution

$$
S_{\lambda}(t)=\phi_{\lambda}(t) S_{\lambda}(0)+\int_{0}^{t} d t_{1} \frac{\phi_{\lambda}(t)}{\phi_{\lambda}\left(t_{1}\right)}\left[\beta h_{\lambda}+\beta g \Psi_{\lambda}(0) E\left(t_{1}\right)+\xi_{\lambda}\left(t_{1}\right)\right] .
$$

The correlation function

$$
C\left(t, t^{\prime}\right)=\frac{1}{N} \sum_{\lambda}\left\langle S_{\lambda}(t) S_{\lambda}\left(t^{\prime}\right)\right\rangle_{a v}
$$

must satisfy the equal time relation $C(t, t)=1$ at all times in view of the spherical condition (10). From Eqs. (9)-(10) with the aid of Eq. (3) we thus find

$$
\begin{aligned}
1= & \left\langle\left\langle\phi_{\lambda}(t)^{2} S_{\lambda}(0)^{2}\right\rangle_{0}+2 \int_{0}^{t} d t_{1}\left\langle\left\langle\frac{\phi_{\lambda}(t)^{2}}{\phi_{\lambda}\left(t_{1}\right)^{2}}\right\rangle_{0}+\beta^{2} \Delta \int_{0}^{t} d t_{1} \int_{0}^{t} d t_{2}\left\langle\left\langle\frac{\phi_{\lambda}(t)^{2}}{\phi_{\lambda}\left(t_{1}\right) \phi_{\lambda}\left(t_{2}\right)}\right\rangle_{0}\right.\right.\right. \\
& +\beta^{2} g^{2} \int_{0}^{t} d t_{1} \int_{0}^{t} d t_{2}\left\langle\frac{\phi_{\lambda}(t)^{2}}{\phi_{\lambda}\left(t_{1}\right) \phi_{\lambda}\left(t_{2}\right)}\right\rangle E\left(t_{1}\right) E\left(t_{2}\right) .
\end{aligned}
$$

This is an implicit equation for the Lagrange multiplier $z(t)$. The two types of averages are defined as

$$
\begin{gathered}
\left\langle\left\langle f_{\lambda}\right\rangle\right\rangle_{0} \equiv \frac{1}{N} \sum_{\lambda} f_{\lambda}=\int d J_{\lambda} \rho_{0}\left(J_{\lambda}\right) f\left(J_{\lambda}\right) ; \\
\left\langle\left\langle f_{\lambda}\right\rangle\right\rangle \equiv \frac{1}{N} \sum_{\lambda} \Psi_{\lambda}(0)^{2} f_{\lambda}=\int d J_{\lambda} \rho\left(J_{\lambda}\right) f\left(J_{\lambda}\right),
\end{gathered}
$$

where $\rho_{0}\left(J_{\lambda}\right)$ and $\rho\left(J_{\lambda}\right)$ are the densities of eigenvalues in the $k \neq 0$ and $k=0$ sector of the spectrum, respectively. The eigenvalues $J_{\lambda}$ have a continuous spectrum $-2 J<J_{\lambda}<2 J$. 
If $\left|J_{0}\right|>J$, there is also a discrete eigenvalue at $J_{m}=J_{0}+J^{2} / J_{0}$ [14. Here we will only discuss the case $\left|J_{0}\right|<J$. The $k \neq 0$ density of states is given by the Wigner semicircle law 14,15

$$
\rho_{0}\left(J_{\lambda}\right)=\frac{1}{2 \pi J^{2}}\left(4 J^{2}-J_{\lambda}^{2}\right)^{1 / 2}
$$

The $k=0$ sector, on the other hand, has the density [15]

$$
\rho\left(J_{\lambda}\right)=\frac{1}{\left.2 \pi\left(J^{2}+J_{0}^{2}-J_{0} J\right) \lambda\right)}\left(4 J^{2}-J_{\lambda}^{2}\right)^{1 / 2}
$$

This density of states has a statistical weight $\mathcal{O}(1 / N)$ and is thus relevant only in averages containing factors of the type $\Psi_{\lambda}(0)^{2} \propto \mathcal{O}(N)$.

The dielectric polarization of the system can be expressed in terms of the solution (9) as

$$
P(t)=\frac{1}{N} \sum_{\lambda} g \Psi_{\lambda}(0) \phi_{\lambda}(t) S_{\lambda}(0)+\beta g^{2} \int_{0}^{t} d t_{1}\left\langle\left\langle\frac{\phi_{\lambda}(t)}{\phi_{\lambda}\left(t_{1}\right)}\right\rangle E\left(t_{1}\right) .\right.
$$

As shown by Cugliandolo and Dean [13], for times larger than a limiting time $t_{c}$ the system in which $\Delta \neq 0$ will always reach an equilibrium state and will thus be characterized by equilibrium dynamics. All information about the initial state $S_{\lambda}(0)$ is lost for $t \gg t_{c}$, i.e., the first term in Eq. (16) becomes irrelevant. In the present case, $t_{c}$ is estimated as $t_{c} \approx 2 \tau J T / \Delta$. Typically, the asymptotic regime $t \gg t_{c}$ is explored in a dielectric relaxation experiment. In the following, we will limit ourselves to this regime. Also, for simplicity we will henceforth set $g=1$.

\section{STATIC DIELECTRIC RESPONSE}

We first consider the case of a static electric field $E(t)=E$ applied at $t=0$. At asymptotic times $t / \tau \gg 1$ the system reaches equilibrium and the Lagrange multiplier $z(t)$ tends to a constant value $z$. Thus the function (8) becomes

$$
\phi_{\lambda}(t) \sim e^{-g_{\lambda} t} ; \quad\left(g_{\lambda} \equiv 2 z-\beta J_{\lambda}\right)
$$

and we can evaluate the integrals in Eqs. (11) and (16). Assuming that $2 z>\beta J_{\lambda}$ for all $\lambda$ (to be justified later) we derive the equation for $z$ :

$$
1=\left\langle\left\langle\frac{1}{2 z-\beta J_{\lambda}}\right\rangle_{0}+\beta^{2} \Delta\left\langle\left\langle\frac{1}{\left(2 z-\beta J_{\lambda}\right)^{2}}\right\rangle_{0}+\beta^{2} E^{2}\left\langle\left\langle\frac{1}{\left(2 z-\beta J_{\lambda}\right)^{2}}\right\rangle .\right.\right.\right.
$$

The static linear susceptibility $\chi_{1}=(\partial P / \partial E)_{E=0}$ is derived from Eqs. (16) and (17):

$$
\chi_{1}=\beta\left\langle\left\langle\frac{1}{g_{\lambda}}\right\rangle\right. \text {. }
$$

The averages in Eqs. (18) and (19) can be expressed in terms of the generalized averages obtained by adding an imaginary generating field $i y$ to the variable $g_{\lambda}$, namely, 


$$
\chi_{1}^{[n]}(y) \equiv \beta\left\langle\frac{1}{\left(g_{\lambda}-i y\right)^{(n+1)}}\right\rangle .
$$

These averages can be evaluated with the aid of Eqs. (12)-(15) for $n=0$, differentiating $n$ times with respect to $i y$, and setting $y=0$. For example, from Eqs. (19) and (20) with $n=0$ and $y=0$ we find:

$$
\chi_{1}=\chi_{1}^{[0]}(0)=\frac{z-r-\beta J_{0}}{\beta\left(J^{2}+J_{0}^{2}\right)-2 \beta J_{0} z},
$$

where $r \equiv \sqrt{z^{2}-\beta^{2} J^{2}}$.

The $n=1$ average is given by

$$
\chi_{1}^{[1]}(y)=\frac{\beta}{2} \frac{z(y)-r(y)}{D(y)}\left[\frac{1}{r(y)}-\frac{2 \beta J_{0}}{D(y)}\right]+\frac{\beta^{3} J_{0}^{2}}{D(y)^{2}},
$$

where $z(y) \equiv z-i y / 2, r(y) \equiv \sqrt{z(y)^{2}-\beta^{2} J^{2}}$, and $D(y) \equiv \beta^{2}\left(J^{2}+J_{0}^{2}\right)-2 \beta J_{0} z(y)$.

The above equation for $z$, Eq. (18), becomes in this notation:

$$
1=\frac{1}{\beta} \chi_{1}^{[0]}(0)_{0}+\beta \Delta \chi_{1}^{[1]}(0)_{0}+\beta E^{2} \chi_{1}^{[1]}(0)
$$

where the averages $\chi_{1}^{[0]}(0)_{0}$ and $\chi_{1}^{[1]}(0)_{0}$ are obtained by setting $J_{0}=0$ in Eqs. (21) and (22), respectively, and $\chi_{1}^{[1]}(0)$ is given by Eq. (22) with $y=0$.

We will also need the $n=2$ average

$$
\chi_{1}^{[2]}(0)_{0}=\frac{1}{8 \beta J^{2}}\left[\frac{z^{2}}{r^{3}}-\frac{1}{r}\right] .
$$

A numerical solution $z(T)$ of Eq. (23) in zero field $(E=0)$ can be found at all temperatures and is independent of $J_{0}$ as long as $\left|J_{0}\right|<J$. An example is shown on Fig. 1 for $\Delta / J^{2}=0.001$. The inset shows that $z-\beta J$ is always positive, and since $2 J$ is the largest eigenvalue of $J_{\lambda}$ one can see that indeed $2 z>2 \beta J_{\lambda}$ for all $\lambda$ as assumed earlier. When both $E \neq 0$ and $J_{0} \neq 0$, there are in general two complex solutions for $z(E, T)$ and the present theory is not applicable. In the following we will only consider the cases in which a real solution $z(T)$ exists and has a real second derivative $z^{\prime \prime}=d^{2} z / d E^{2}$ at $E=0$.

For $\Delta=0$ and $E=0$, Eq. (23) reduces to the equation derived in Ref. [14, which has the solution $z_{0}=\left(1+\beta^{2} J^{2}\right) / 2$ for $T \geq J$. For $T<J$, however, the solution does not exist and $z$ must be obtained from the saddle-point condition [14], yielding $z_{0}=\beta J$.

A numerical evaluation shows that the expression (21) for the static linear susceptibility fully agrees with the result obtained by means of replica theory in Ref. [7].

One can also calculate the static third-order nonlinear susceptibility $\chi_{3}$, which is defined in terms of the expansion

$$
P=\chi_{1} E-\chi_{3} E^{3}+\cdots .
$$

Obviously, $\chi_{3}=-(1 / 6)\left(\partial^{3} P / \partial E^{3}\right)_{E=0}$. Using Eqs. (16), (17) and (20) we find 


$$
\chi_{3}=\chi_{1}^{[1]}(0) z_{0}^{\prime \prime}
$$

Evaluating $z_{0}^{\prime \prime} \equiv\left(d^{2} z / d E^{2}\right)_{E=0}$ from Eq. (23) we obtain the result

$$
\chi_{3}=\beta^{2} \frac{\chi_{1}^{[1]}(0)^{2}}{\chi_{1}^{[1]}(0)_{0}+2 \beta^{2} \Delta \chi_{1}^{[2]}(0)_{0}},
$$

where $\chi_{1}^{[2]}(0)_{0}$ is given by Eq. (24) above. The last expression has been evaluated numerically and found to be precisely equivalent to the result derived in Ref. [7] using the replica formalism.

\section{DYNAMIC RESPONSE}

We now consider the case of an oscillating electric field $E(t)=E_{0} \cos (\omega t)$. This is inserted into Eq. (16). At asymptotic times $t \gg t_{c}$ the response can be written by analogy with Eq. (25) as 16

$$
P(t) \sim\left[P_{\omega} e^{-i \omega t}+P_{3 \omega} e^{-i 3 \omega t}+\cdots\right]+\text { c.c. },
$$

where $P_{\omega}$ and $P_{3 \omega}$ are the amplitudes of the first and third harmonic response, respectively, which are given by

$$
\begin{gathered}
P_{\omega}=\chi_{1,0}(\omega)\left(\frac{E_{0}}{2}\right)+\chi_{1,1}(\omega)\left(\frac{E_{0}}{2}\right)^{3}+\cdots ; \\
P_{3 \omega}=\chi_{3,0}(\omega)\left(\frac{E_{0}}{2}\right)^{3}+\chi_{3,1}(\omega)\left(\frac{E_{0}}{2}\right)^{5}+\cdots .
\end{gathered}
$$

Here we have introduced the linear dynamic response $\chi_{1,0}(\omega)$, the third-order nonlinear responses $\chi_{1,1}(\omega)$ and $\chi_{3,0}(\omega)$, etc. We will focus on the first harmonic linear response $\chi_{1,0}(\omega)$, which is equivalent to the dynamic linear susceptibility $\chi_{1}(\omega)=\chi_{1,0}(\omega)$, and on the third-order nonlinear response $\chi_{3,0}(\omega)$. The latter is typically measured by monitoring the third harmonic component of $P(t)$ at small amplitudes of the field $E_{0}$ [19]. In order to ensure the proper static limit $\omega \rightarrow 0$ we will define the third-order nonlinear dynamic susceptibility as $\chi_{3}(\omega)=-\chi_{3,0}(\omega)$. From Eqs. (28)-(30) we thus find

$$
\begin{gathered}
\chi_{1}(\omega)=\left.\frac{\partial P_{\omega}}{\partial\left(E_{0} / 2\right)}\right|_{E_{0}=0} ; \\
\chi_{3}(\omega)=-\left.\frac{1}{6} \frac{\partial^{3} P_{3 \omega}}{\partial\left(E_{0} / 2\right)^{3}}\right|_{E_{0}=0} .
\end{gathered}
$$

In the asymptotic regime, the function $\phi_{\lambda}(t)$ in Eq. (16) behaves as

$$
\phi_{\lambda}(t) \sim e^{-g_{\lambda} t-2 \varphi(t)},
$$


where we have defined $\varphi(t) \equiv \int_{0}^{t} d t^{\prime}\left[z\left(t^{\prime}\right)-z\right]$, with $z$ representing the solution of Eq. (18). The first part of the response (16), which will be proportional to $\sim E_{0} \exp (-i \omega t)$, is now given by

$$
P(t)=\beta\left(\frac{E_{0}}{2}\right) \int_{0}^{t} d t_{1}\left\langle\left\langle e^{-g_{\lambda}\left(t-t_{1}\right)}\right\rangle e^{-2\left[\varphi(t)-\varphi\left(t_{1}\right)\right]} e^{-i \omega t_{1}}+\right.\text { c.c. }
$$

\section{A. Linear dynamic susceptibility}

The part of $P_{1}(\omega)$, which is linear in $E_{0}$, is trivially obtained from Eq. (34) by noting that $\varphi(t)=0$ for $E_{0}=0$. We can thus evaluate the integral and using Eq. (31) we find

$$
\chi_{1}(\omega)=\beta\left\langle\left\langle\frac{1}{g_{\lambda}-i \omega}\right\rangle .\right.
$$

Comparing with Eqs. (19) and (20) we realize that the averages of the above type can be evaluated with the aid of Eq. (20), in which we set $y=\omega$ and $n=0$, yielding (with $\tau$ restored)

$$
\chi_{1}(\omega)=\frac{z-i \omega \tau / 2-\sqrt{(z-i \omega \tau / 2)^{2}-\beta^{2} J^{2}}-\beta J_{0}}{\beta\left(J^{2}+J_{0}^{2}\right)-2 J_{0}(z-i \omega \tau / 2)} .
$$

For $\omega \rightarrow 0$ this obviously reduces to the static susceptibility (21).

The temperature behavior of $\chi_{1}(\omega)$ will crucially depend on the temperature variation of the relaxation time $\tau(T)$. The SRBRF model (2) and the equations of motion (1) contain no information about $\tau(T)$. It has been found empirically [17,2,6] that some of the properties of relaxors can be described by assuming a Vogel-Fulcher (VF) relationship for $\tau$, namely,

$$
\tau=\tau_{0} \exp \left(\frac{U}{T-T_{0}}\right)
$$

where $T_{0}$ is the VF temperature. This expression is valid for $T>T_{0}$ and would lead to a divergence of $\tau$ for $T \rightarrow T_{0}$. There is no a priori relation between $T_{0}$ and the parameters of the SRBRF model. A similar situation occurs in Ising dipolar glasses, where a probability distribution of relaxation times $g(\ln \tau)$ has been used in combination with an empirical Debye-type response [18]. With $\tau$ lying in the range $\tau_{\min }<\tau<\tau_{\max }$, the VF temperature $T_{0}$ has been identified with the freezing temperature $T_{f}$. On the other hand, $\tau_{\min }$ has been fitted to an Arrhenius-type expression $\tau_{\text {min }} \propto \exp (E / T)$. The same approach was found to be applicable to relaxors as well [19.

An alternative approach is based on the master equation for the reorientation of cluster polarization assuming a VF relaxation time of the type (37), where the barrier heights $U$ are distributed according to a Gaussian probability function [2,6]. Such an approach was found to be applicable to PMN and PST in the region $T>T_{0}$.

In general, we can thus introduce the average dynamic susceptibility

$$
\bar{\chi}_{1}(\omega)=\int_{\tau_{\min }}^{\tau_{\max }} \frac{d \tau}{\tau} g(\ln \tau) \chi_{1}(\omega)
$$


where the probability distribution of relaxation times $g(\ln \tau)$ is physically justified by the fact that relaxors are inherently inhomogeneous systems due to compositional disorder. Thus one may imagine, for example, that the relaxor system consists of a set of macroscopic regions, which are formally characterized by the same microscopic equation of motion, but differ in the value of the parameter $\tau$.

One encounters serious difficulties in attempting to describe the dynamic response at $T<$ $T_{0}$. Formally one could assume that $\tau \rightarrow \infty$ for $T \leq T_{0}$, but this will lead to a zero value of $\chi_{1}(\omega)=0$ at all temperatures $T \leq T_{0}$. We can single out the following representative cases: (i) a single VF relaxation time (37); (ii) a nonsingular distribution of barrier heights $g(U)$; (iii) a distribution of relaxation times $g(\ln \tau)$ such that its normalization $\int_{\tau_{\min }}^{\tau_{\max }} d \tau g(\ln \tau) / \tau$ diverges as $\tau_{\max } \rightarrow \infty$. The first case is illustrated in Fig. 2, where we show the calculated real and imaginary parts of $\chi_{1}(T, \omega)=0$ for several values of frequency assuming a single VF relaxation time (37). As in Fig. 1 we assume $J_{0}=0.9 J$ and $\Delta / J^{2}=0.001$, as well as $T_{0}=J$. Such behavior is incompatible with experiments, which generally show a smooth decrease of $\chi_{1}^{\prime}(T, \omega)$ and $\chi_{1}^{\prime \prime}(T, \omega)$ across the region where $T_{0}$ is expected to be located.

A more realistic description can be obtained, for example, by assuming a distribution $w\left(T_{0}\right)$ of VF temperatures $T_{0}$, where $T_{0}$ is allowed to vary in the range $0<T_{0}<T_{0}^{\max }$. Using the relation $d(\ln \tau) g(\ln \tau)=d T_{0} w\left(T_{0}\right)$ in Eq. (38) and choosing a linear distribution $w\left(T_{0}\right)=2\left(1-T_{0} / J\right) / J$ with $T_{0}^{\max }=J$, we obtain the temperature dependence of the linear susceptibility shown in Fig. 3. Here we used the same set of parameters as in Fig. 2. In contrast to the single VF temperature case, the above distribution leads to nonzero values of $\bar{\chi}_{1}(\omega)$ at all temperatures. The shape of the real and imaginary part of $\bar{\chi}_{1}(\omega)$ is in qualitative agreement with the observed relaxation spectra in PMN [19] and PLZT [20].

It should be noted, however, that the above result for the linear susceptibility contains only the contribution of polar clusters. Other contributions may exist-for example, that of optic phonons - which are not expected to show any anomalies near $T_{f}$. In general, such contributions can be written as a sum of Debye-like terms, with the possibility of an average over the corresponding relaxation times. At present, the problem of a realistic relaxation time distribution in relaxors, which would be appropriate at all temperatures, has not yet been resolved.

\section{B. Zero-field-cooled susceptibility}

In analogy with Ising spin glasses [21] and dipolar glasses [22] one can introduce an effective relaxation time for the low-frequency response

$$
\tau_{e f f}=-i \beta \lim _{\omega \rightarrow 0} \frac{\partial \chi_{1}(\omega)^{-1}}{\partial \omega} .
$$

Returning to Eq. (31) and using the above definition of $\chi_{1}^{[1]}(y)$ from Eq. (22) in which we set $y=\omega \tau$, we obtain the result

$$
\tau_{e f f}=\frac{1}{2} \beta \tau \lim _{\omega \rightarrow 0} \frac{\chi_{1}^{[1]}(\omega \tau)}{\chi_{1}(\omega)^{2}} .
$$

It will be shown later that the real part of the nonlinear susceptibility $\chi_{3}(\omega)$ is also proportional to $\chi_{1}^{[1]}(\omega)$ and in the static limit shows a sharp peak at the "freezing" temperature 
$T_{f} \approx J$ for $\Delta \ll J^{2}$, while it actually diverges if $\Delta=0$. Since $\chi_{1}(\omega)$ is a well behaved function of temperature, it follows that $\tau_{\text {eff }}$ increases as $\sim(T-J)^{-1}$ on approaching $T_{f}$, however, it remains finite at $T_{f}$. Thus the behavior of $\tau_{\text {eff }}$ mimics the freezing transition in this case. A true freezing transition at $T_{f}=T_{0}$ can be described by assuming a VF temperature dependence of $\tau$, which is then transferred to $\tau_{\text {eff }}$ via Eq. (40).

When $\tau_{\text {eff }}$ is large, the system will only reach equilibrium at times $t \gg \tau_{\text {eff }}$, which may become very long. In measuring the static susceptibility $\chi_{1}$ one should distinguish between the field-cooled and zero-field-cooled susceptibility, $\chi_{1}^{F C}$ and $\chi_{1}^{Z F C}$, respectively. Here $\chi_{1}^{F C}$ is given by Eq. (19) and corresponds to an experiment carried out on a time scale $t \gg \tau_{\text {eff }}$. On the other hand, $\chi_{1}^{Z F C}$ can be obtained by turning on a field $E\left(t_{1}\right)=E$ at time $t_{1}=0$ and measuring the response $P(t)$ at time $t>t_{1}$ as described by Eq. (16). Thus we can write

$$
\chi_{1}^{Z F C}(t)=\frac{\partial P(t)}{\partial E}=\beta \int_{0}^{t} d t_{1}\left\langle\frac{\phi_{\lambda}(t)}{\phi_{\lambda}\left(t_{1}\right)}\right\rangle,
$$

and using Eq. (17) we find

$$
\chi_{1}^{Z F C}(t)=\beta\left\langle\frac{1-e^{-\left(2 z-\beta J_{\lambda}\right) t / \tau}}{2 z-\beta J_{\lambda}}\right\rangle .
$$

For $t \rightarrow \infty$ this reduces to the previous result (19), which corresponds to $\chi_{1}^{F C}$. The difference between the two susceptibilities $\delta \chi_{1}(t) \equiv \chi_{1}^{F C}-\chi_{1}^{Z F C}(t)$ is given by

$$
\delta \chi_{1}(t)=\beta\left\langle\frac{e^{-\left(2 z-\beta J_{\lambda}\right) t / \tau}}{2 z-\beta J_{\lambda}}\right\rangle=\beta \int_{t / \tau}^{\infty} d t_{1}\left\langle\left\langle e^{-\left(2 z-\beta J_{\lambda}\right) t_{1}}\right\rangle .\right.
$$

The value of the integral can be estimated by first using Eqs. (13) and (15) and expanding the integrand in powers of $J_{0} / J$ [23]. To leading order the result is independent of the parameter $J_{0}$ and shows that for $\Delta \ll J^{2}$ and $T \leq J$ one has a power law behavior $\delta \chi_{1}(t) \sim(t / \tau)^{-1 / 2}$, implying a slow decay and a large difference between the two susceptibilities, which has been observed experimentally [19]. On the other hand, for $T>J$ the asymptotic behavior is a combination of power law and exponential, i.e., $\delta \chi_{1}(t) \sim(t / \tau)^{-3 / 2} \exp [-2(z-\beta J) t / \tau]$. Thus in this regime the difference decays much faster and the two susceptibilities become indistinguishable on a typical experimental time scale.

\section{NONLINEAR DIELECTRIC SUSCEPTIBILITY}

To calculate the third order partial derivative in Eq. (32) we return to Eq. (34), in which $\varphi(t)$ is now a function of $E_{0}$. In general, $\varphi(t)$ will be a sum of terms, which are even powers of $E_{0} e^{ \pm i \omega t}$. We will focus on the second-order term $\sim E_{0}^{2} e^{-2 i \omega t}$. Introducing the function

$$
X(t)=\left.\frac{\partial^{2} \varphi(t)}{\partial\left(E_{0} / 2\right)^{2}}\right|_{E_{0}=0}
$$

we can express the third partial derivative in Eq. (32) as

$$
\left.\frac{\partial^{3} P_{3}(\omega)}{\partial\left(E_{0} / 2\right)^{3}}\right|_{E_{0}=0}=-6 \beta e^{2 i \omega t} \int_{0}^{t} d t_{1} e^{i \omega\left(t-t_{1}\right)}\left\langle\left\langle e^{-g_{\lambda}\left(t-t_{1}\right)}\right\rangle\left[X(t)-X\left(t_{1}\right)\right] .\right.
$$


The function $X(t)$ will be calculated from Eq. (11) in the asymptotic limit. Considering only the terms, which asymptotically behave as $\sim e^{-2 i \omega t}$ and taking the second derivative with respect to $E_{0} / 2$ leads to

$$
\begin{aligned}
0= & -4\left[\int _ { 0 } ^ { t } d t _ { 1 } \left\langle\left\langle e^{-2 g_{\lambda}\left(t-t_{1}\right)}\right\rangle_{0}-2 \beta^{2} \Delta \int_{0}^{t} d t_{1} \int_{0}^{t} d t_{2}\left\langle\left\langle e^{-2 g_{\lambda}\left(2 t-t_{1}\right)-t_{2}}\right\rangle_{0}\right]\left[X(t)-X\left(t_{1}\right)\right]\right.\right. \\
& +\beta^{2} \int_{0}^{t} d t_{1} \int_{0}^{t} d t_{2}\left\langle\left\langle e^{-2 g_{\lambda}\left(2 t-t_{1}-t_{2}\right.}\right\rangle e^{-i \omega\left(t_{1}+t_{2}\right)} .\right.
\end{aligned}
$$

The last double integral becomes for asymptotic values of $t$

$$
+\beta^{2} e^{-2 i \omega t}\left\langle\frac{1}{\left(g_{\lambda}-i \omega\right)^{2}}\right\rangle
$$

We now apply the Laplace transform to Eq. (46) using the definition

$$
\tilde{X}(p)=\int_{0}^{\infty} d t e^{-p t} X(t)
$$

and obtain the result:

$$
\tilde{X}(p)=\frac{\beta^{2}}{2(p+2 i \omega)} \frac{\left\langle\left\langle\frac{1}{\left(g_{\lambda}-i \omega\right)^{2}}\right\rangle\right\rangle}{\left\langle\left\langle\frac{1}{g_{\lambda}}\right\rangle\right\rangle_{0}-\left\langle\left\langle\frac{1}{g_{\lambda}+p / 2}\right\rangle\right\rangle_{0}+\beta^{2} \Delta\left[\left\langle\left\langle\frac{1}{g_{\lambda}^{2}}\right\rangle\right\rangle_{0}-\left\langle\left\langle\frac{1}{g_{\lambda}\left(g_{\lambda}+p\right)}\right\rangle\right\rangle_{0}\right]} .
$$

The averages can be expressed in terms of the generalized responses (22).

The function $X(t)$ can be obtained by the inverse Laplace transform. Its behavior is determined by the poles $p_{k}=p_{k}^{\prime}+i p_{k}^{\prime \prime}$ of $\tilde{X}(p)$. A numerical evaluation shows that all poles are such that $p_{k}^{\prime} \leq 0$. At asymptotic times only those poles for which $p_{k}^{\prime}=0$ will be relevant. There is only one such pole, namely, $p_{0}=-2 i \omega$ leading to

$$
X(t) \sim\left(\frac{\beta^{2}}{2}\right) \frac{\chi_{1}^{[1]}(\omega) e^{-2 i \omega t}}{\chi_{1}(0)_{0}-\chi_{1}(\omega)_{0}+\beta^{2} \Delta\left[\chi_{1}^{[1]}(0)_{0}+\frac{\chi_{1}(0)_{0}-\chi_{1}(2 \omega)_{0}}{2 i \omega}\right]} .
$$

Inserting this expression into Eq. (45), evaluating the integral, and returning to Eq. (32), we obtain the final result for the complex third-order nonlinear susceptibility (with $\tau$ restored):

$$
\chi_{3}(\omega)=\left(\frac{\beta^{2}}{2}\right) \frac{\chi_{1}^{[1]}(\omega \tau)\left[\chi_{1}(\omega)-\chi_{1}(3 \omega)\right]}{\chi_{1}(0)_{0}-\chi_{1}(\omega)_{0}+\beta^{2} \Delta\left[\chi_{1}^{[1]}(0)_{0}+\frac{\chi_{1}(0)_{0}-\chi_{1}(2 \omega)_{0}}{2 i \omega \tau}\right]} .
$$

Here $\chi_{1}^{[1]}(\omega \tau)$ is given by Eq. (22]) with $y=\omega \tau$, and $\chi_{1}(\omega)$ by Eq. (36).

This expression may now be averaged over the distribution of relaxation times $\tau$ as argued in the paragraph preceding Eq. (39). In Fig. 4 the real and imaginary parts of $\bar{\chi}_{3}(\omega)$ are plotted as functions of temperature for several values of frequency. In analogy with the case of $\bar{\chi}_{1}(\omega)$ above, a VF behavior (37) of $\tau$ and a linear distribution of VF temperatures $T_{0}$ has been used. The values of the parameters are again $J_{0} / J=0.9$ and $\Delta / J^{2}=0.001$. 
The real part $\bar{\chi}_{1}^{\prime}(T, \omega)$ has a sharp peak near $T \simeq J$, whose origin can be traced back to the function $\chi_{1}^{[1]}(\omega)$ appearing in Eq. (51). As in the linear susceptibility case, a strong frequency dispersion is evident. In the limit of small frequencies, i.e., $\omega \tau_{0} \ll 1$, the behavior of $\bar{\chi}_{3}^{\prime}(T)$ is the same as in the static case studied by replica theory [7]. At high frequencies and low temperatures, $\bar{\chi}_{3}^{\prime}(T, \omega)$ may become negative due to the last factor in the numerator of Eq. (51), whose imaginary part changes sign. This effect cannot be observed easily, since this would require a measurement of the nonlinear susceptibility in the range where the absolute value of $\bar{\chi}_{3}^{\prime}(T, \omega)$ is extremely small compared to its peak value.

It is easily verified that in the limit $\omega \rightarrow 0, \mathrm{Eq}$. (51) reduces to the static result (27).

It has been shown in the static theory [7] that a crucial quantity, which can discriminate between the dipolar glass-like and ferroelectric behavior, is not $\chi_{3}(T)$ but rather the rescaled static nonlinear susceptibility $a_{3}=\chi_{3} / \chi_{1}^{4}$. In spin glasses without random fields $a_{3}(T)$ diverges at $T_{f}$, and in a relaxor it develops a peak near $T_{f}$, whereas in a ferroelectric with long range order $a_{3}$ decreases with decreasing temperature on approaching the critical temperature $T_{c}$ [19]. It has been suggested that $a_{3}(T)$ could also be extracted from the dynamic linear and nonlinear susceptibilities by considering the following generalized function [16,24]:

$$
a_{3}^{\prime}(T, \omega)=\frac{\bar{\chi}_{3}^{\prime}(\omega)}{\bar{\chi}_{1}^{\prime}(3 \omega) \bar{\chi}_{1}^{\prime}(\omega)^{3}} .
$$

In Fig. $5, a_{3}^{\prime}(T, \omega)$ is plotted as a function of temperature for the same set of parameters as in Fig. 4. Each of the three factors in Eq. (52) has been averaged over the linear distribution of $T_{0}$. Obviously, $a_{3}^{\prime}(T, \omega)$ develops a peak near $T_{f} \simeq J$ at all frequencies shown. On the high- $T$ side of the peak, $a_{3}^{\prime}(T)$ is independent of frequency and agrees with the static $a_{3}$. Near the peak and on its low- $T$ side, however, strong frequency dispersion appears. Recent experiments in PMN and PLZT [5] indicate that the high- $T$ quasistatic part of $a_{3}(T)$ exhibits a crossover between the paraelectric-like decreasing behavior and a glass-like increasing behavior on approaching $T_{f}$. This type of behavior is characteristic of relaxor ferroelectrics. The crossover can be qualitatively described by the present dynamic SRBRF model, as shown in the inset of Fig. 5. The model also correctly predicts the onset of strong frequency dispersion in $a_{3}^{\prime}(T, \omega)$ at low temperatures, which has been observed in both PMN and PLZT [5].

\section{CONCLUSIONS}

We have presented a dynamic model of uniaxial relaxor ferroelectrics based on the recently developed static spherical random-bond-random-field (SRBRF) model [7]. Following the theory of spherical models of spin glasses [12,13 the order parameter field is assumed to obey the Langevin equation of motion written in the representation of eigenstates of the random interaction matrix with the spherical condition being enforced at all times. The equations of motion are exactly solvable in the asymptotic limit where the relaxor system reaches an equilibrium state. The linear and the third-order nonlinear dynamic response functions have been derived. In the static $\omega \rightarrow 0$ limit these results are precisely equivalent to the static linear and nonlinear susceptibilities $\chi_{1}$ and $\chi_{3}$, respectively, obtained earlier by the replica method [7]. 
In analogy with the static case, the dynamic theory does not predict a sharp transition into a dipolar glass-like state. Rather, in the case of weak random fields the third-order susceptibility shows a narrow peak at a temperature $T_{f}$, which mimics the freezing transition. Within the context of a dynamic model the freezing transition would correspond to the divergence of the longest relaxation time in the system. However, the dynamic SRBRF model contains no information on the behavior of the relaxation time $\tau$ appearing in the equations of motion, and does not lead to the divergence of the effective relaxation time on approaching $T_{f}$. In order to describe the observed freezing transition one should therefore introduce a divergent behavior of $\tau$. This can be done, for example, by assuming a VogelFulcher (VF) law for the temperature behavior of $\tau$ in accordance with empirical findings [2],6], however, this will suppress the dynamic response at all temperatures lower than the VF temperature $T_{0}$. We have shown that by introducing a probability distribution of VF temperatures $T_{0}$ one can obtain linear and nonlinear response functions which remain finite at all temperatures, in qualitative agreement with experiments. The largest value of $T_{0}$ has been set equal to the static "freezing" temperature $T_{f}$, which is determined by the random bond strength parameter $J$.

The actual shape of $\bar{\chi}_{1}(T, \omega)$ and $\bar{\chi}_{3}(T, \omega)$, where the bar means an average over $\tau$ or equivalently over $T_{0}$, strongly depends on the probability distribution of relaxation times $g(\ln \tau)$.

Within the framework of the dynamic SRBRF model we have also calculated the scaled dynamic nonlinear susceptibility $a_{3}^{\prime}(T, \omega)=\bar{\chi}_{3}^{\prime}(\omega) / \bar{\chi}_{1}^{\prime}(3 \omega) \bar{\chi}_{1}^{\prime}(\omega)^{3}$, which allows one to discriminate between the ferroelectric-like and glass-like behavior of relaxors [5,24]. In the quasistatic regime above $T_{f}, a_{3}^{\prime}(T, \omega)$ is practically independent of $\omega$ and its temperature dependence shows a crossover between paraelectric-like and glass-like behavior on approaching $T_{f}$ from above. This crossover behavior has recently been observed both in PMN and PLZT [5]. The calculated shape of $\bar{\chi}_{1}(T, \omega)$ and $\bar{\chi}_{3}(T, \omega)$, and hence of $a_{3}^{\prime}(T, \omega)$, strongly depends on the probability distribution of relaxation times $g(\ln \tau)$. In the present work we $\operatorname{did}$ not attempt to investigate in detail the effects of $g(\ln \tau)$ on the behavior of these quantities; however, we have shown that if one assumes a linear distribution of VF temperatures $T_{0}$, the predicted behavior of $\bar{\chi}_{1}(T, \omega)$ and $\bar{\chi}_{3}(T, \omega)$ is in qualitative agreement with experiments in PMN [5, 19,24] and PLZT [20,5]. On the other hand, $a_{3}^{\prime}(T, \omega)$ obtained in this manner has a peak near $T_{f} \simeq J$ and shows a strong frequency dispersion below $T_{f}$. The predicted peak in $a_{3}^{\prime}(T, \omega)$ has not been observed experimentally [24], suggesting that one should perhaps search for a more realistic distribution $g(\ln \tau)$. This problem will be dealt with in a future publication.

\section{ACKNOWLEDGMENTS}

This work was supported by the Ministry for Science and Technology of the Republic of Slovenia. One of the authors (R. P.) is grateful to Silvio Franz and to Boris E. Vugmeister for helpful suggestions. 


\section{REFERENCES}

[1] L. E. Cross, Ferroelectrics 76, 241 (1987); 151, 305 (1994).

[2] B. E. Vugmeister and H. Rabitz, Phys. Rev. B 57, 7581 (1998).

[3] R. Blinc, J. Dolinšek, A. Gregorovič, B. Zalar, C. Filipič, Z. Kutnjak, A. Levstik, and R. Pirc, Phys. Rev. Lett. 83, 424 (1999).

[4] R. Blinc, A. Gregorovič, B. Zalar, R. Pirc, and S.G. Lushnikov, Phys. Rev. B 61, 253 (2000).

[5] V. Bobnar, Z. Kutnjak, R. Pirc, R. Blinc, and A. Levstik, Phys. Rev. Lett. 84, 5892 (2000).

[6] B. E. Vugmeister and H. Rabitz, Phys. Rev. B 61, 14448 (2000).

[7] R. Pirc and R. Blinc, Phys. Rev. B 60, 13470 (1999).

[8] V. Bobnar, Z. Kutnjak, R. Pirc, and A. Levstik, Phys. Rev. B 60, 6420, (1999).

[9] V. Westphal, W. Kleemann, and M. D. Glinchuk, Phys. Rev. Lett. 68, 847 (1992).

[10] R. Pirc, B.Tadić, and R. Blinc, Phys. Rev. B 36, 8607 (1987).

[11] N. Korner, Ch. Pfammatter, and R. Kind, Phys. Rev. Lett. 70, 1283 (1993).

[12] L.F. Cugliandolo and D. S. Dean, J. Phys. A: Math. Gen. 28, 4213 (1995).

[13] L.F. Cugliandolo and D. S. Dean, J. Phys. A: Math. Gen. 28, L453 (1995).

[14] J. Kosterlitz, D. Thouless, and R.C. Jones, Phys. Rev. Lett. 36, 1217 (1976).

[15] D.C. Mattis, Spin Glasses (Springer-Verlag, Berlin, 1985), Chap. 2.12.

[16] Y. Ishibashi, J. Korean Phys. Soc. 32, S407 (1998).

[17] G.A. Samara, Ferroelectrics 117, 347 (1991); Phys. Rev. Lett. 77, 314 (1996).

[18] Z. Kutnjak, R. Pirc, A. Levstik, I. Levstik, C. Filipič, R. Blinc, and R. Kind, Phys. Rev. B 50, 12421 (1994).

[19] A. Levstik, Z. Kutnjak, C. Filipič, and R. Pirc, Phys. Rev. B 57, 11204 (1998).

[20] Z. Kutnjak, C. Filipič, R. Pirc, A. Levstik, R. Farhi, and M. El Marssi, Phys. Rev. B 59, 294 (1999).

[21] H. Sompolinsky and A. Zippelius, Phys. Rev. B 25, 6860 (1982).

[22] B.Tadić, R. Pirc, and R. Blinc, Z. Phys. B 75, 249 (1989).

[23] R. Pirc (unpublished).

[24] A.E. Glazounov, Ph. D. Thesis No. 1665, EPFL, Lausanne (1997); A.E. Glazounov and A.K. Tagantsev, Phys. Rev. Lett. 85, 2192 (2000). 


\section{FIGURES}

FIG. 1. Solution of Eq. (23) for the Lagrange multiplier $z(T)$ obtained numerically in zero field $(E=0)$ and for $\Delta / J^{2}=0.001$. Inset: Proof that $z-\beta J>0$ at all temperatures.

FIG. 2. Real and imaginary parts of the linear susceptibility in the case of a single Vogel-Fulcher (VF) type relaxation time [Eq. (37)] as functions of temperature for several values of frequency, as indicated. Note that the response is strictly zero below the VF temperature $T_{0}=J$.

FIG. 3. Real and imaginary parts of the linear susceptibility averaged over a linear probability distribution of VF temperature $T_{0}$, with $0<T_{0}<J$.

FIG. 4. Calculated temperature dependence of the real and imaginary parts of the third-order nonlinear susceptibility averaged over a linear distribution of $T_{0}$, using the same parameter values as in Figs. 2 and 3.

FIG. 5. Temperature dependence of the scaled nonlinear susceptibility

$a_{3}^{\prime}(T, \omega)=\bar{\chi}_{3}^{\prime}(\omega) / \bar{\chi}_{1}^{\prime}(3 \omega) \bar{\chi}_{1}^{\prime}(\omega)^{3}$ obtained with the same parameter values as in Figs. 2 - 4 . The inset shows the crossover from the decreasing paraelectric-like to increasing glass-like behavior in the quasistatic regime above the peak. 
Pirc et al., Fig. 1

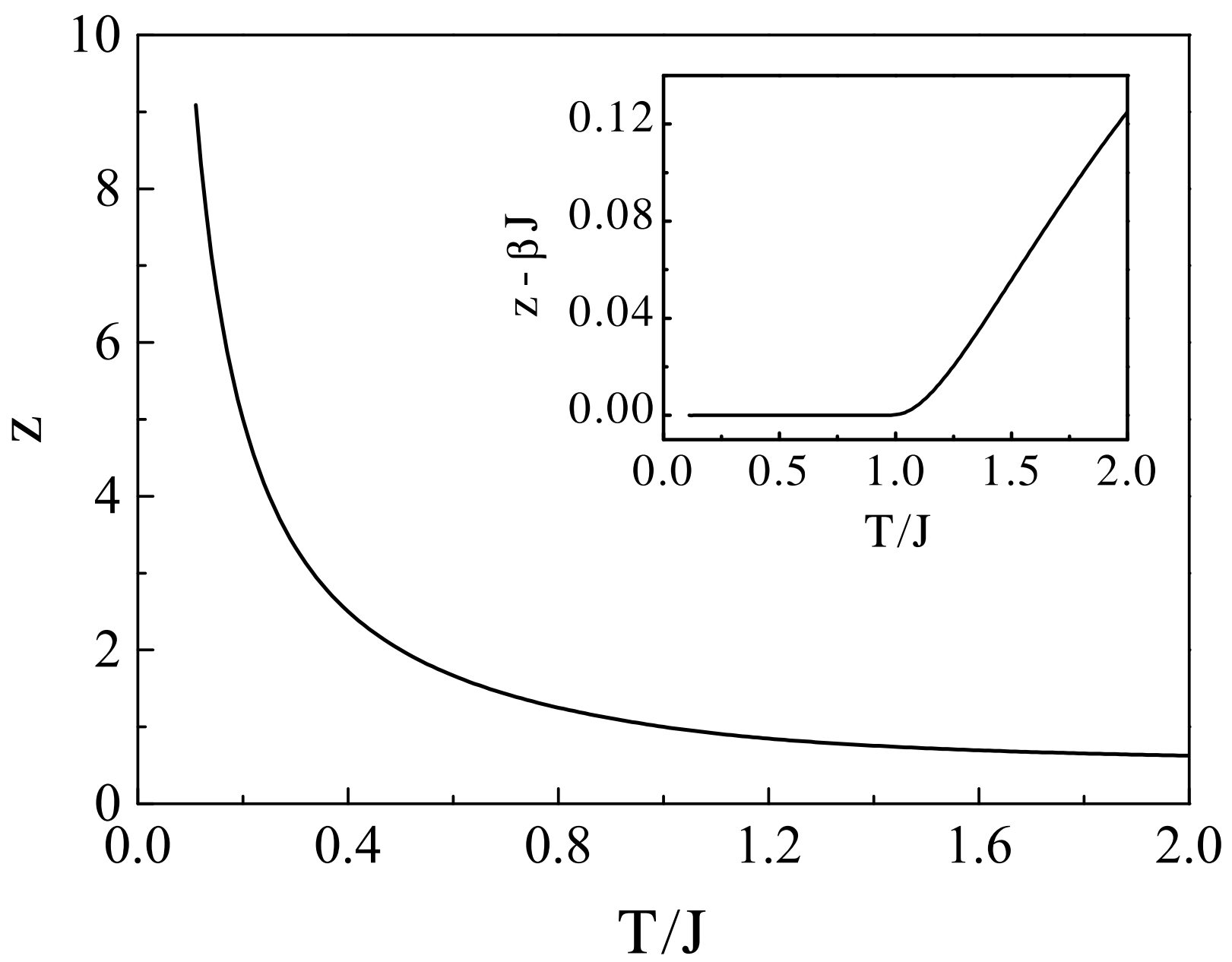


Pirc et al., Fig. 2

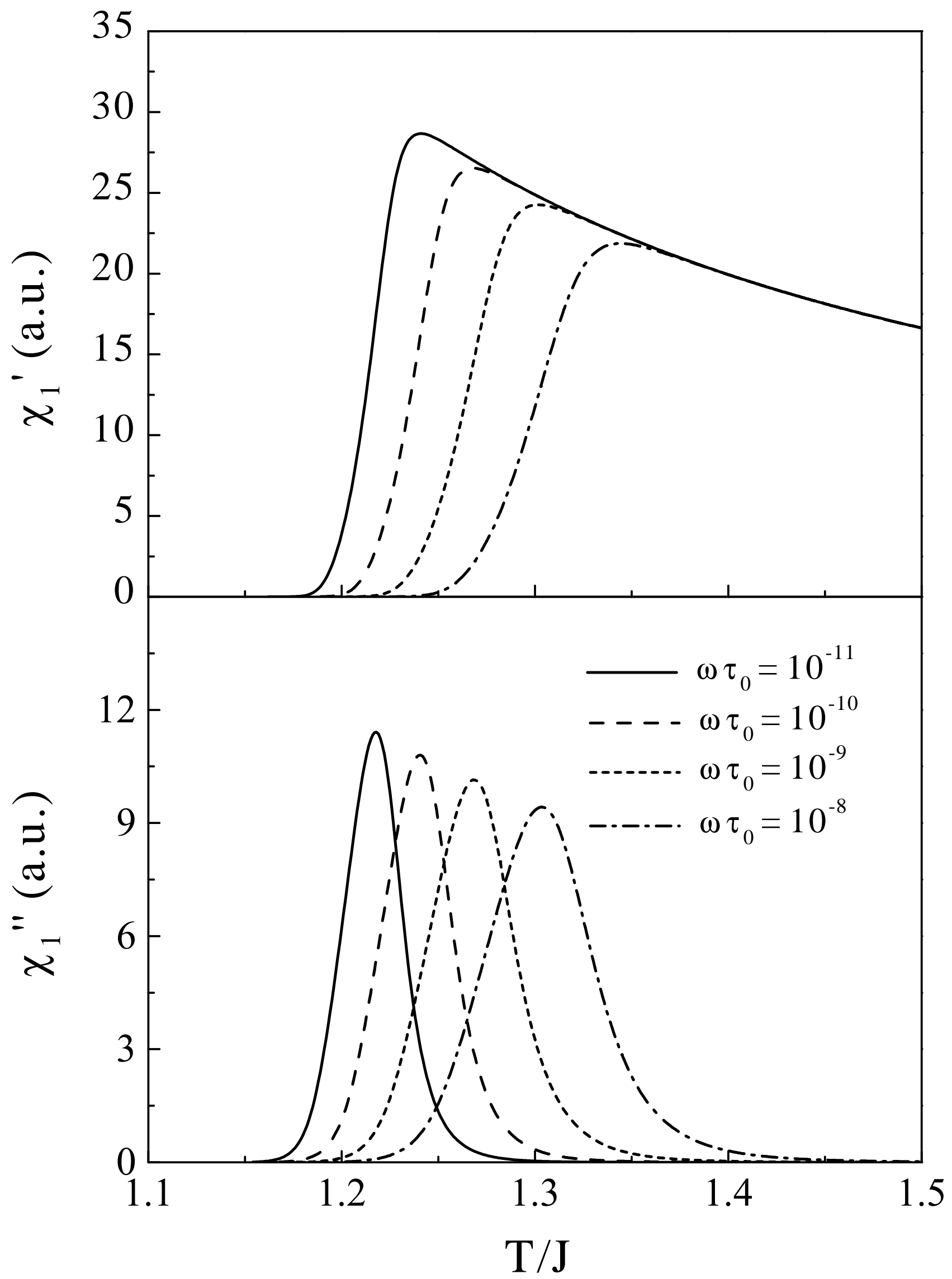


Pirc et al., Fig. 3

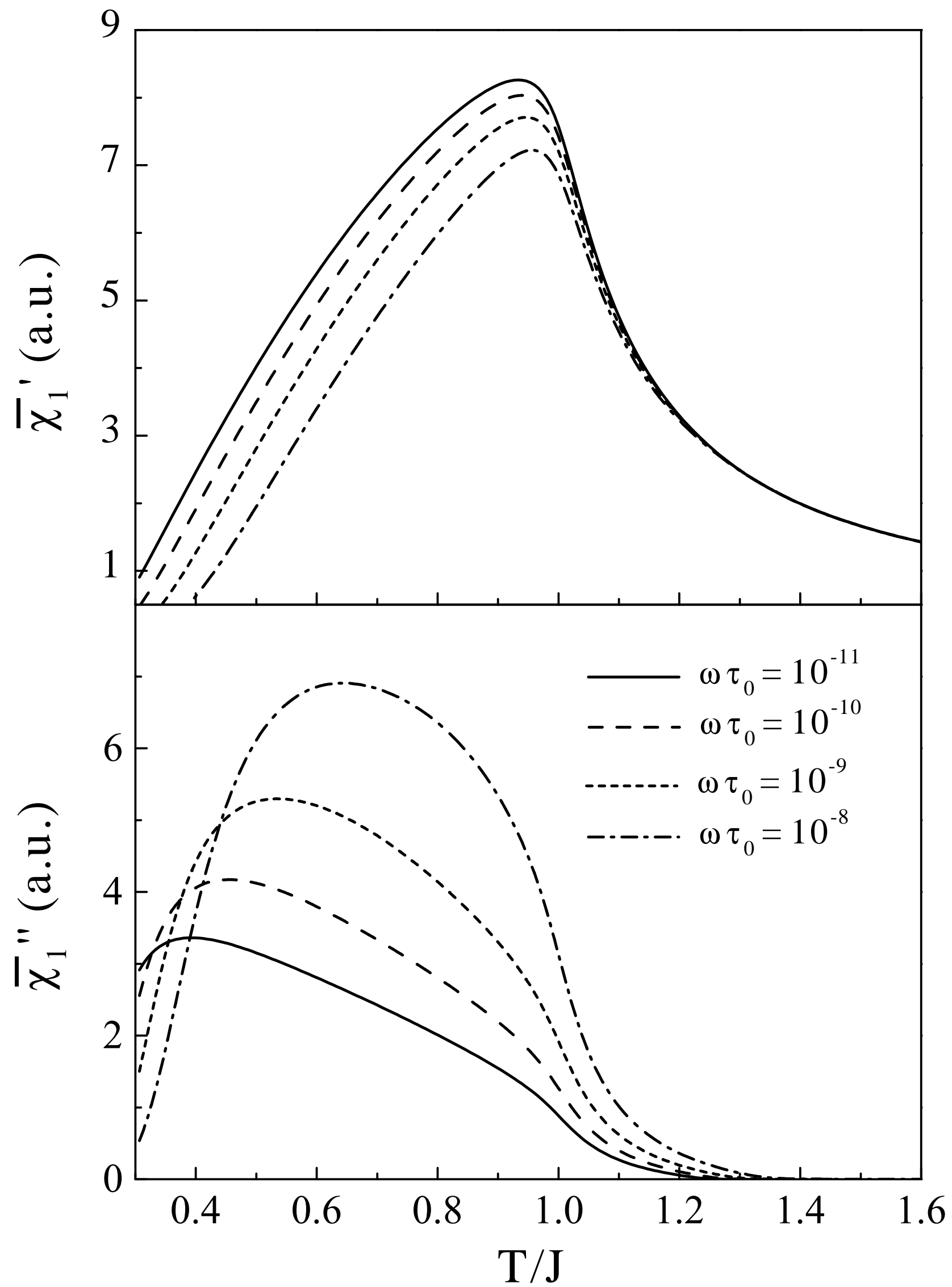


Pirc et al., Fig. 4

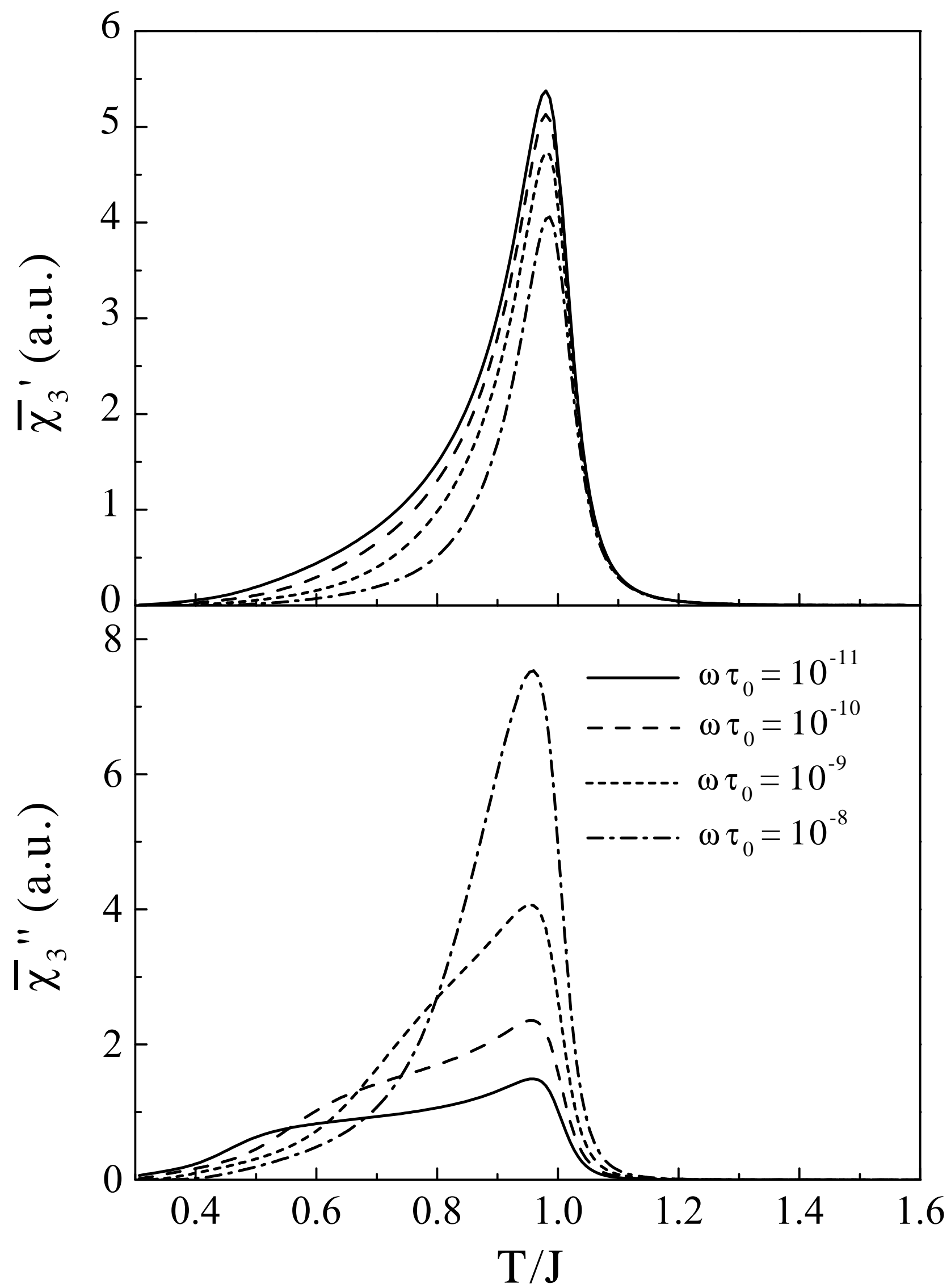


Pirc et al., Fig. 5

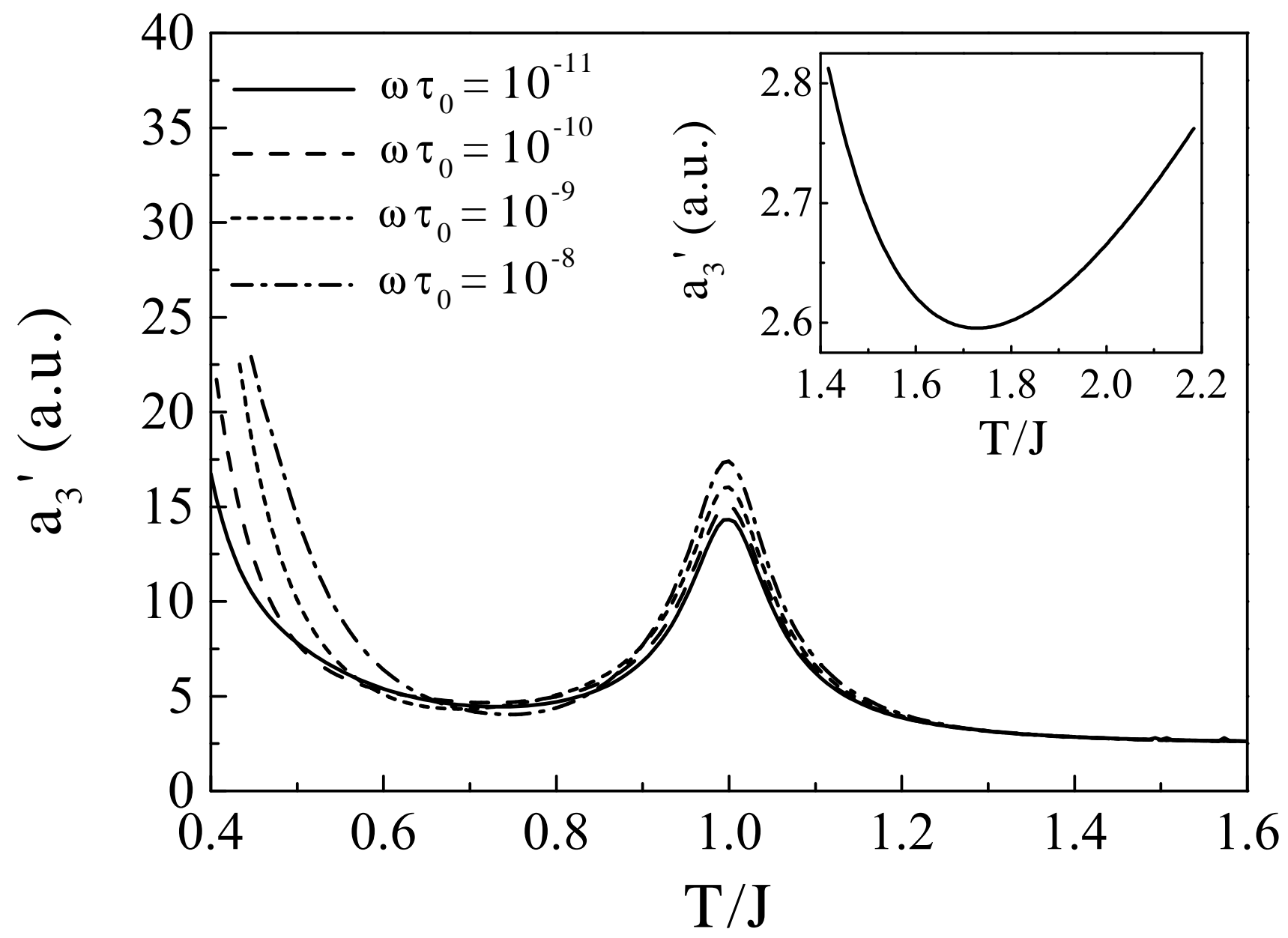

